\title{
Urticaria por frío: serie de casos y revisión del tema
}

\author{
Jorge Mario Sánchez ${ }^{1,2}$, Ruth Helena Ramírez¹, Liliana María Tamayo, \\ Carlos Fernando Chinchilla ${ }^{1}$, Ricardo Cardona ${ }^{1}$ \\ 1 Grupo de Alergología Clínica y Experimental (GACE), Universidad de Antioquia, Medellín, Colombia \\ 2 Fundación para el Desarrollo de las Ciencias Médicas y Biológicas (FUNDEMEB), Cartagena, Colombia \\ La urticaria por frío es una de las cinco causas más frecuentes de urticarias crónicas; está incluida \\ en el grupo de urticarias físicas y puede ocurrir luego de la exposición al frío, ya sea a través de \\ un objeto, el aire o un líquido. Los pacientes pueden presentar síntomas de urticaria, angioedema, \\ dificultad respiratoria e, incluso, anafilaxia al sacar objetos de la nevera, nadar en agua fría o al entrar \\ a cuartos con aire acondicionado. A continuación se presentan cinco pacientes con diagnóstico de esta \\ enfermedad y una breve revisión del tema.
}

Palabras clave: urticaria, angioedema, anafilaxia, antagonistas de histamina, crioglobulinemia, frío.

\section{Cold urticaria: case series and literature review}

Cold urticaria is one of the five most common causes of chronic urticaria and is grouped as a physical urticaria. It can occur after exposure to cold, either through solid objects, air or liquids. Patients may have symptoms of urticaria, angioedema, respiratory distress and even anaphilaxis when the skin is exposed to a cold environment, such as handling refrigerated objects, swimming in cold water or entering an air-conditioned room. Five cases of cold urticaria are presented, followed by a brief literature review.

Key words: Urticaria, angioedema, anaphylaxis, histamine antagonists, cryoglobulinemia, cold temperatura.

\section{Caso 1}

Se trata de un paciente de sexo masculino de 41 años de edad, que consultó por un cuadro clínico de dos meses de evolución consistente en urticaria, angioedema en manos y pies, y dificultad respiratoria cuando estaba en una piscina, que cedió espontáneamente; también había presentado urticaria en un antebrazo al contacto con una botella fría. Tenía antecedentes personales de rinitis, asma y conjuntivitis alérgica. Se le practicó la prueba del cubo de hielo, que fue positiva a los cuatro minutos. Fue tratado con fexofenadina, con mejoría total de los síntomas.

\section{Caso 2}

Se trata de una paciente de sexo femenino de 3 años y 8 meses de edad, que fue llevada a consulta por presentar urticaria y angioedema al contacto con el agua fría. En una ocasión, mientras estaba

\section{Correspondencia:}

Jorge Mario Sánchez, Servicio de Alergología Clínica, Clínica León XIII, bloque I, consultorios 111, 112 y 113, Medellín, Colombia.

Teléfonos: (574) 516 7300, extensión 3455; celular: (300) 808 4249

jotamsc@yahoo.com

Recibido: 01/06/10; aceptado:10/02/11 en una piscina, presentó un episodio de hipotonía generalizada, palidez y pérdida de conciencia, que requirió hospitalización. Con el consumo de bebidas frías presentaba eritema y edema en cuello. Se le practicó la prueba del cubo de hielo, que fue positiva a los seis minutos. Se inició tratamiento con ciproheptadina, consiguiéndose mejoría de la sintomatología. Un mes después del inicio del tratamiento, se hizo una nueva prueba con cubo de hielo, la cual fue negativa a los 10 minutos. La paciente recibió ciproheptadina por seis meses llegando hasta la dosis tope para su peso, pero por persistencia de síntomas asociados al contacto con el frío, se decidió cambiar el tratamiento por cetirizina más montelukast. La paciente ha sido seguida durante cuatro años y en la actualidad todavía presenta urticaria asociada al baño y en piscinas, pero sin afectación respiratoria y de resolución espontánea.

\section{Caso 3}

Se trata de un paciente de sexo masculino de 41 años de edad. Consultó por un año de evolución de urticaria generalizada desencadenada por el baño frío, y urticaria localizada en áreas expuestas a bebidas frías y lavado de manos con agua fría. También, presentaba síntomas cuando se exponía a la lluvia o a un ambiente frío. Los 
habones duraban 10 minutos, aproximadamente, y desaparecían espontáneamente. No había tenido afectación respiratoria. Se hizo la prueba del cubo de hielo, que fue positiva a los tres minutos. No había antecedentes personales de enfermedades atópicas. Se inició tratamiento con desloratadina y se logró el control de los síntomas.

\section{Caso 4}

Se trata de una paciente de sexo femenino de 26 años de edad, que consultó por un cuadro clínico de cinco meses de evolución consistente en pápulas eritematosas, prurito intenso y angioedema asociado al contacto con el frío y, en algunas ocasiones, asociado a disnea. Los síntomas se presentaban con ambiente frío o con agua fría y sucedían en el área expuesta. Cuando se bañaba en piscina o con agua fría, la reacción era generalizada; cuando lavaba platos o se lavaba las manos, la reacción era local. Cuando ingería bebidas frías, no presentaba edema en lengua ni disfagia, pero sí reacción en las manos al sostener la botella o el vaso frío. La paciente había recibido loratadina y cetirizina, y mejoró del prurito pero no del resto de los síntomas. No había antecedentes personales ni familiares de enfermedades atópicas. Se hizo la prueba del cubo de hielo que fue positiva al minuto. Se inició tratamiento con $5 \mathrm{mg}$ al día de desloratadina y $10 \mathrm{mg}$ al día de montelukast Tres meses después fue revisada nuevamente y refirió tolerar el consumo de bebidas frías y el contacto con agua y ambientes fríos. Continúa con los medicamentos.

\section{Caso 5}

Se trara de una paciente de sexo femenino de 14 años de edad, que consultó por un cuadro clínico de tres meses de evolución consistente en urticaria asociada al baño con agua fría y a la inmersión en piscina, y también había presentado angioedema en labios con el consumo de helados. No se había asociado con dificultad respiratoria. No había antecedentes personales de enfermedades atópicas, pero sí el antecedente familiar de un hermano con rinitis alérgica $y$, en el padre, asma y urticaria por frío en la infancia. Se practicó la prueba del cubo de hielo, que fue positiva a los cinco minutos. Se inició tratamiento con desloratadina y montelukast, y se logró control de los síntomas pocas semanas después.

\section{Introducción}

La urticaria por frío es una forma frecuente de urticaria física caracterizada por una reacción cutánea de tipo habón, angioedema o ambos, después del contacto con un estímulo frío (1). Parece afectar más a las mujeres que a los hombres y su incidencia cambia dependiendo del clima en la región geográfica, encontrándose incidencias desde $5 \%$ en clima cálido y hasta de $30 \%$ en zonas con clima templado $(2,3)$.

Las implicaciones que tiene en la calidad de vida de las personas afectadas hacen de su diagnóstico y tratamiento una prioridad para el médico tratante. Este tipo de urticaria se ha relacionado con el antecedente de atopia en aproximadamente $45 \%$ de los pacientes. En un estudio con 56 pacientes, $45 \%$ tenía este antecedente (4). Actualmente, la urticaria por frío se divide en adquirida o familiar. A continuación, se describen en detalle las urticarias por frío, adquiridas, primarias, que son las más frecuentes $(5,6)$ (cuadro 1).

\section{Diagnóstico y clasificación}

Como su nombre lo indica, la urticaria por frío se presenta cuando el paciente se expone a temperaturas frías que pueden ser producidas por el clima, el agua, las bebidas o el contacto con objetos que estén a bajas temperaturas. El resultado puede ser solamente urticaria o asociarse con angioedema e, incluso, presentarse con dificultad respiratoria y choque anafiláctico. El umbral que la desencadena varía entre las personas y los síntomas, que pueden variar desde urticaria sola o con angioedema y hasta con dificultad respiratoria o choque anafiláctico (caso 5). Suele presentarse minutos después del contacto, pero, en algunas ocasiones, tarda horas o días.

Las herramientas diagnósticas han cambiado poco desde 1840, cuando Bourdon publicó las primeras descripciones de pacientes, y se basa en dos pilares que son la historia clínica y el reto cutáneo del paciente. Esta prueba ha tenido modificaciones, pero básicamente consiste en observar la formación de un habón luego de un estímulo frío en relación con el tiempo (7-9). Se denomina "test del cubo de hielo" y consiste en colocar un cubo de hielo $(0 \mathrm{a}$ $4{ }^{\circ} \mathrm{C}$ ) sobre el antebrazo por cinco minutos, luego retirarlo y esperar, aproximadamente, 10 minutos para que la piel recupere su temperatura normal.

Se considera que la prueba es positiva cuando hay formación de un habón o de habones confluentes en el lugar de la aplicación. Se recomienda cubrir el cubo de hielo con una bolsa plástica para evitar el contacto de la piel con el agua y, de esta manera, no confundir el diagnóstico con una 
Cuadro 1. Exámenes de laboratorio disponibles por cada paciente al momento de iniciar el tratamiento.

\begin{tabular}{llllll}
\hline Exámenes de laboratorio & \multicolumn{5}{c}{ Paciente } \\
\cline { 2 - 6 } & \multicolumn{1}{c}{$\mathbf{1}$} & $\mathbf{2}$ & $\mathbf{3}$ & $\mathbf{4}$ & $\mathbf{5}$ \\
\hline Hemoglobina (g/dl) & 16,5 & 11,3 & 15,8 & 14,3 & 14,7 \\
Hematocrito (\%) & 48,9 & 35,1 & 45,1 & 45,2 & 43,8 \\
Leucocitos (total de células) & 6.370 & 7.200 & 5.290 & 9.310 & 6.430 \\
Polimorfonucleares (\%) & 2.548 & 3.744 & 2.645 & 6.657 & 2.870 \\
Linfocitos (\%) & 2.802 & 2.808 & 1.957 & 1.517 & 2.630 \\
Eosinófilos (\%) & 446 & 72 & 159 & 233 & 350 \\
Basófilos (\%) & 63 & 144 & 53 & 19 & 20 \\
THS (IU/ml) & 4,05 & -- & 4,83 & Negativas & Negativas \\
Crioaglutininas & -- & Negativas & Negativas & 108,6 & 103,2 \\
C3 (mg/dl) & -- & 127 & -- & 21,4 & 18 \\
C4 (mg/dl) & -- & 35 & -- & Negativo & -- \\
ANA & Negativo & Negativo & -- & No reactivo & -- \\
VDRL & No reactiva & -- & No reactivo & Negativo & -- \\
Factor reumatoideo & Negativo & Negativo & -- & & \\
\hline
\end{tabular}

THS: tirotropina; ANA: anticuerpos antinucleares; VDRL: Venereal Disease Research Laboratory

urticaria acuagénica (caso 5). Si hay formación del habón, se procede a producir estímulos más cortos con reducciones de un minuto, hasta encontrar el tiempo mínimo con el cual reacciona el paciente (casos 3 y 4). Estas nuevas exposiciones deben hacerse en diferentes sitios del antebrazo, para evitar resultados negativos por degranulación de los mastocitos y desensibilización de la piel.

Si en la primera exposición de cinco minutos no hay formación de habón, se aumenta el tiempo de exposición en un minuto (caso 2), generalmente hasta diez minutos; si en ninguna de estas nuevas exposiciones hay formación de habón, se considera que la prueba es negativa y es poco probable que el paciente tenga urticaria por frío. Se ha observado que entre menor es el tiempo de exposición que desencadena la reacción, mayor es el riesgo de presentar reacciones sistémicas ante la exposición por frío, en especial, cuando el habón se forma ante estímulos menores de tres minutos (8) (figura 1).

En la literatura científica se considera que la prueba del cubo de hielo tiene una sensibilidad del $83 \%$ y una especificidad del $100 \%$ (10)

Con la intención de identificar el umbral al cual el paciente presenta los síntomas, algunos investigadores han utilizado otros dispositivos, como Temp Test, basado en el efecto Peltier, que ha demostrado tener igual sensibilidad y especificidad que la prueba del cubo de hielo. Este dispositivo utiliza como fuente de frío el punto intermedio de la temperatura de dos metales y se puede graduar en cuatro valores distintos, lo que permite saber

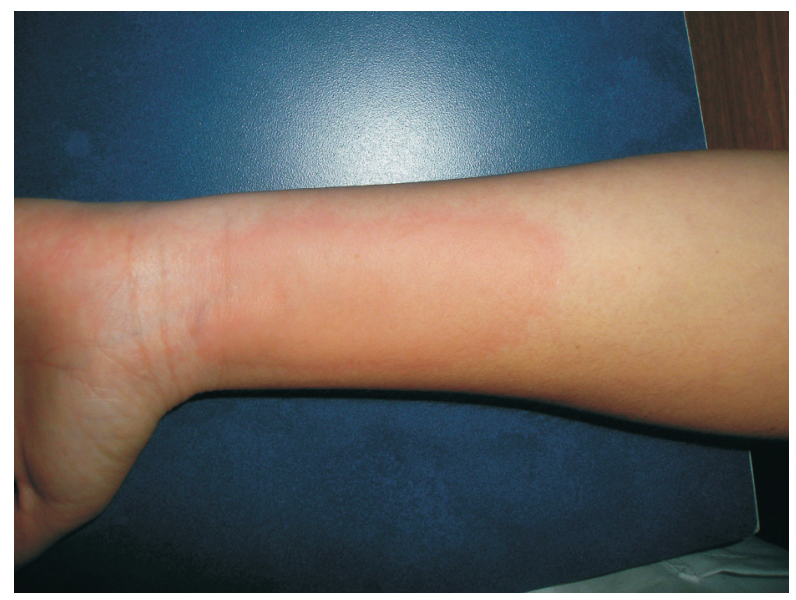

Figura 1. Paciente del caso 4 con prueba de cubo de hielo positiva luego de cinco minutos de exposición.

de manera más exacta la temperatura a la cual el paciente presenta reacción (1).

Otra herramienta diagnóstica es la inmersión de una extremidad (por lo general, la mano) en agua a $10^{\circ} \mathrm{C}$ durante cinco minutos, siguiendo el mismo sistema en el tiempo comentado para la prueba con el cubo de hielo (11). Esta prueba se considera de segunda línea, porque las exposiciones a mayores estímulos fríos, incluso por pocos minutos, aumentan el riesgo de reacciones sistémicas, entre ellas la anafilaxia $(12,13)$.

Con la prueba del cubo de hielo podemos obtener tres resultados: una prueba negativa, una prueba positiva durante el periodo de lectura o una respuesta atípica. 
Las urticarias por frío con respuesta atípica son varias y se encuentran resumidas en el cuadro 2 (14); todas comparten dos características en común:

1) historia clínica de urticaria o angioedema por frío, y

2) prueba del cubo de hielo negativa de manera inmediata (excepto la urticaria por frío refleja).

Algunas infecciones virales (por ejemplo, la mononucleosis, la infección por VIH) (23-25), infecciones bacterianas (borreliosis) e, incluso, por parásitos (Helicobacter pylori, toxoplasma) $(26,27)$ se han implicado como desencadenantes de la urticaria por frío.

Como se observa en la figura 2, las urticarias por frío con prueba del cubo de hielo positiva se dividen en primaria y secundaria. Las primarias explican entre 90 y $95 \%$ de las urticarias adquiridas (13) y para su diagnóstico debe haber una prueba positiva 0 historia clínica de habones o angioedema inducidos por el frío, y al paciente se le deben descartar enfermedades subyacentes que pudieran explicar el cuadro. Los exámenes iniciales de laboratorio incluyen un hemograma completo, velocidad de sedimentación globular, anticuerpos antinucleares, serología para mononucleosis infecciosa, VDRL, fracciones $\mathrm{C} 3$ y C4 del complemento y crioglobulinas. Algunos autores sugieren descartar $\mathrm{VIH}$, debido al reporte de algunos pacientes con esta enfermedad y urticaria por frío.
En la urticaria por frío, secundaria a otras causas, se requiere demostrar una enfermedad subyacente que puede estar causando los síntomas y una prueba positiva. Entre las enfermedades más frecuentemente asociadas están las infecciones previas, como toxoplasmosis (26) o mononucleosis infecciosa (24), y la crioglobulinemia primaria y secundaria (28). Otras enfermedades que se deben descartar son la leucemia linfocítica crónica, la vasculitis leucocitoclástica, la infección por el virus de la hepatitis C y B $(28,29)$, la sífilis, la rubéola y la varicela. La mayoría de los artículos que afirman que las crioglobulinemias son la principal causa de urticaria secundaria se han llevado a cabo con pocos pacientes y tienen más de 50 años de publicación, por lo cual deben ser interpretados con cautela en vista de los cambios actuales en la prevalencia de las enfermedades. La urticaria por frío con respuesta atípica puede presentarse de diferentes maneras, las cuales se resumen en el cuadro 2.

El rango de edad de la población afectada por cualquiera de estas presentaciones es bastante amplio, sin predominio de sexo, a diferencia de las urticarias por frío secundarias que tienen preferencia por el sexo femenino; se han reportado casos de pacientes desde los 3 meses de edad a los 79 años, pero la mayoría de los trabajos muestran que el diagnóstico se hace alrededor de los 20 años, aunque el paciente puede haber prsentado síntomas varios años antes del diagnóstico.

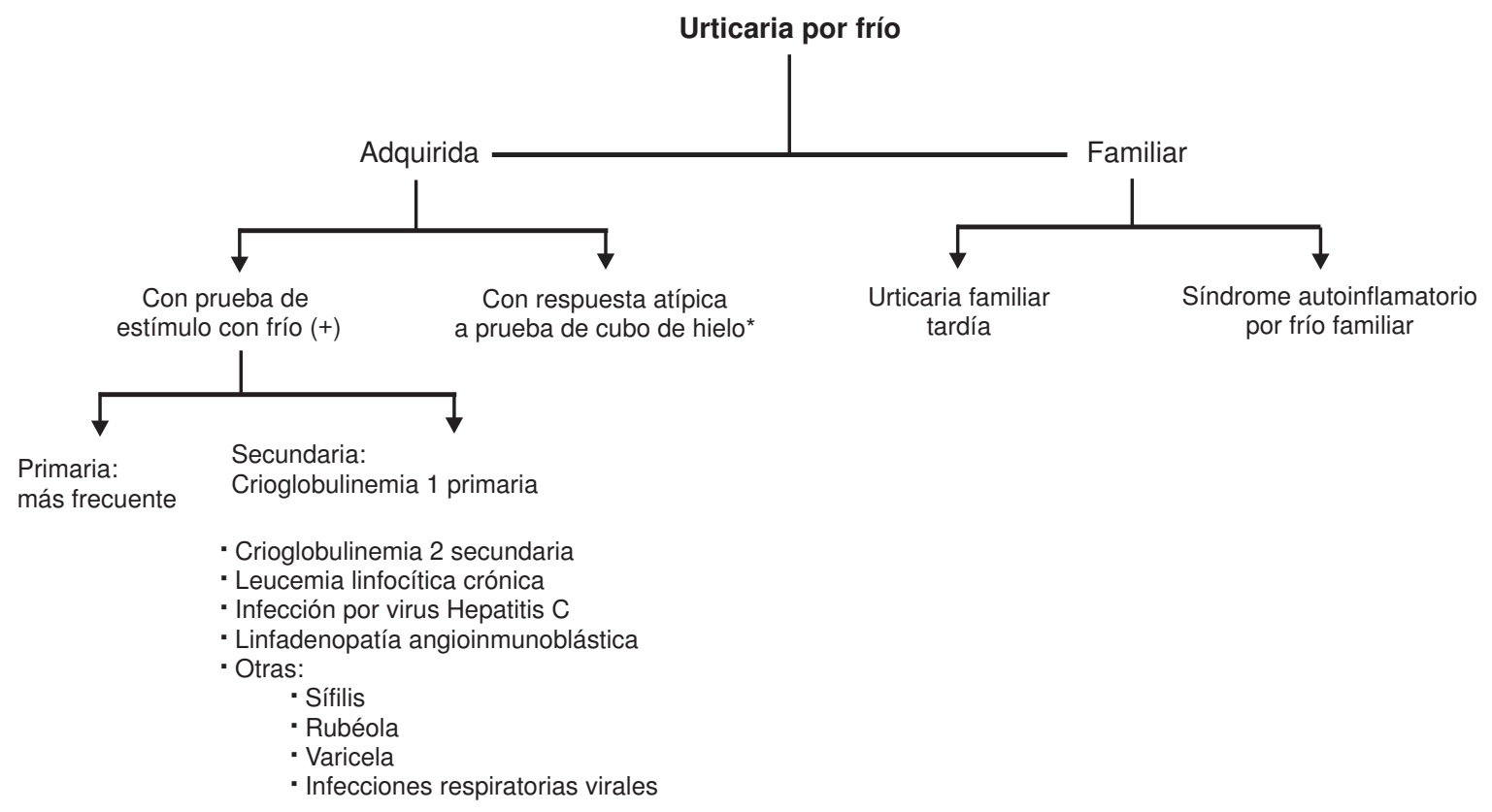

Figura 2. Clasificación de las urticarias por frío. 
Cuadro 2. Urticarias al frío adquiridas atípicas. Todas las urticarias por frío atípicas comparten dos características en común: 1) historia clínica de urticaria o angioedema por frío, y 2) prueba de hielo negativa de manera inmediata, excepto para la urticaria refleja.

\begin{tabular}{|c|c|c|c|}
\hline Urticaria con respuesta atípica & Diagnóstico & Comentario & Referencia \\
\hline Urticaria sistémica atípica & $\begin{array}{l}\text { 1) Síntomas sistémicos con } \\
\text { condiciones ambientales } \\
\text { especiales (por ejemplo, } \\
\text { aire frío húmedo) } \\
\text { 2) Prueba cutánea de cubo } \\
\text { de hielo negativa }\end{array}$ & $\begin{array}{l}\text { Puede desarrollarse } \\
\text { en regiones del cuerpo } \\
\text { no expuestas al frío. }\end{array}$ & $(14-18)$ \\
\hline $\begin{array}{l}\text { Urticaria con dermografismo } \\
\text { dependiente de frío }\end{array}$ & $\begin{array}{l}\text { Inducción de urticaria } \\
\text { luego de frotar la piel fría }\end{array}$ & $\begin{array}{l}\text { Sólo aparece si la piel } \\
\text { estaba fría antes del } \\
\text { frote; con la piel a } \\
\text { temperatura ambiente, no. }\end{array}$ & $(14,18)$ \\
\hline $\begin{array}{l}\text { Urtacaria colinérgica inducida } \\
\text { por frío }\end{array}$ & $\begin{array}{l}\text { 1) El ejercicio en clima frío } \\
\text { lleva a una urticaria } \\
\text { generalizada. } \\
\text { 2) Prueba de metacolina } \\
\text { positiva en la piel }\end{array}$ & $\begin{array}{l}\text { El ejercicio en ambientes } \\
\text { cálidos no produce } \\
\text { síntomas. }\end{array}$ & $(14,19)$ \\
\hline Urticaria al frío tardía & $\begin{array}{l}\text { La urticaria se desarrolla } \\
12 \text { a } 48 \text { horas después } \\
\text { del estímulo frío. }\end{array}$ & $\begin{array}{l}\text { La localización es } \\
\text { preferentemente en } \\
\text { la zona donde hubo } \\
\text { contacto con frío. }\end{array}$ & $(14,20)$ \\
\hline $\begin{array}{l}\text { Urticaria al frío refleja } \\
\text { localizada }\end{array}$ & $\begin{array}{l}\text { La estimulación con frío } \\
\text { produce síntomas inmediatos, } \\
\text { pero en una localización } \\
\text { distante. }\end{array}$ & $\begin{array}{l}\text { Las lesiones se presentan } \\
\text { a una distancia mayor de } \\
5 \mathrm{~cm} \text { del estímulo. }\end{array}$ & $(14,21,22)$ \\
\hline
\end{tabular}

\section{Fisiopatología}

El estudio de la fisiopatología de esta enfermedad ha permitido grandes avances en las mediadas por hipersensibilidad inmediata y, si bien varios de los aspectos que intervienen en su desarrollo aún no están claros, como por qué el frío actúa como agente desencadenante, se han hecho varios avances en el entendimiento de su fisiopatología. El mastocito y su principal mediador, la histamina, han demostrado ser piezas esenciales en esta enfermedad (30) (figura 3).

En los años 50 dos trabajos del grupo de Samson y de Sherman, indicaron que algunos pacientes con urticaria por frío tienen factores en el suero que, al ser aplicados en personas sanas, pueden inducir la enfermedad luego del estímulo frío $(31,32)$.

Estudios posteriores han demostrado que esos factores están asociados con la inmunoglobulina E (lgE) y la inmunoglobulina $M$ (IgM). El descubrimiento de estas inmunoglobulinas llevó a pensar en una respuesta a un antígeno propio por parte de estos anticuerpos, lo que se describió como "la teoría autoinmunitaria".

Actualmente, gracias a los estudios in vitro, se ha demostrado que la inmunoglobulina $G$ ( $(\mathrm{gG}$ ) y la IgM actuarían como los anticuerpos causales de los mecanismos inflamatorios, mientras que la $\mathrm{lgE}$ es el antígeno blanco de la respuesta. Es importante destacar que, a diferencia de la IgG anti-IgE, los anticuerpos IgM anti-lgE sólo se han descrito en pacientes con urticaria por frío y no en otros tipos de urticarias; en los ensayos en los que se ha hecho absorción de lgE y de IgM, se ha observado una menor liberación de histamina. Estos datos apoyarían el hallazgo de que la IgM anti-lgE sería el factor sérico esencial en el desarrollo de la urticaria por frío $(33,34)$.

El por qué se forman dichos anticuerpos y a qué zona de la IgE se estarían fijando, no está claro, pero tal vez su producción se deba a una respuesta inicial a un antígeno exógeno o propio normalmente oculto y, luego, por la similitud de dicho antígeno (aún no identificado) con porciones presentes en la lgE, los anticuerpos formados contra el primer antígeno reaccionarían con esta segunda molécula, incluso luego de haber desaparecido la molécula inicial.

Este tipo de reacción se conoce como mimetismo molecular y se ha demostrado como un mecanismo importante en otras enfermedades $(35,36)$. Pacientes con enfermedades infecciosas, como por ejemplo una mononucleosis $(24,37)$, o que han estado expuestos a situaciones que resulten en una 


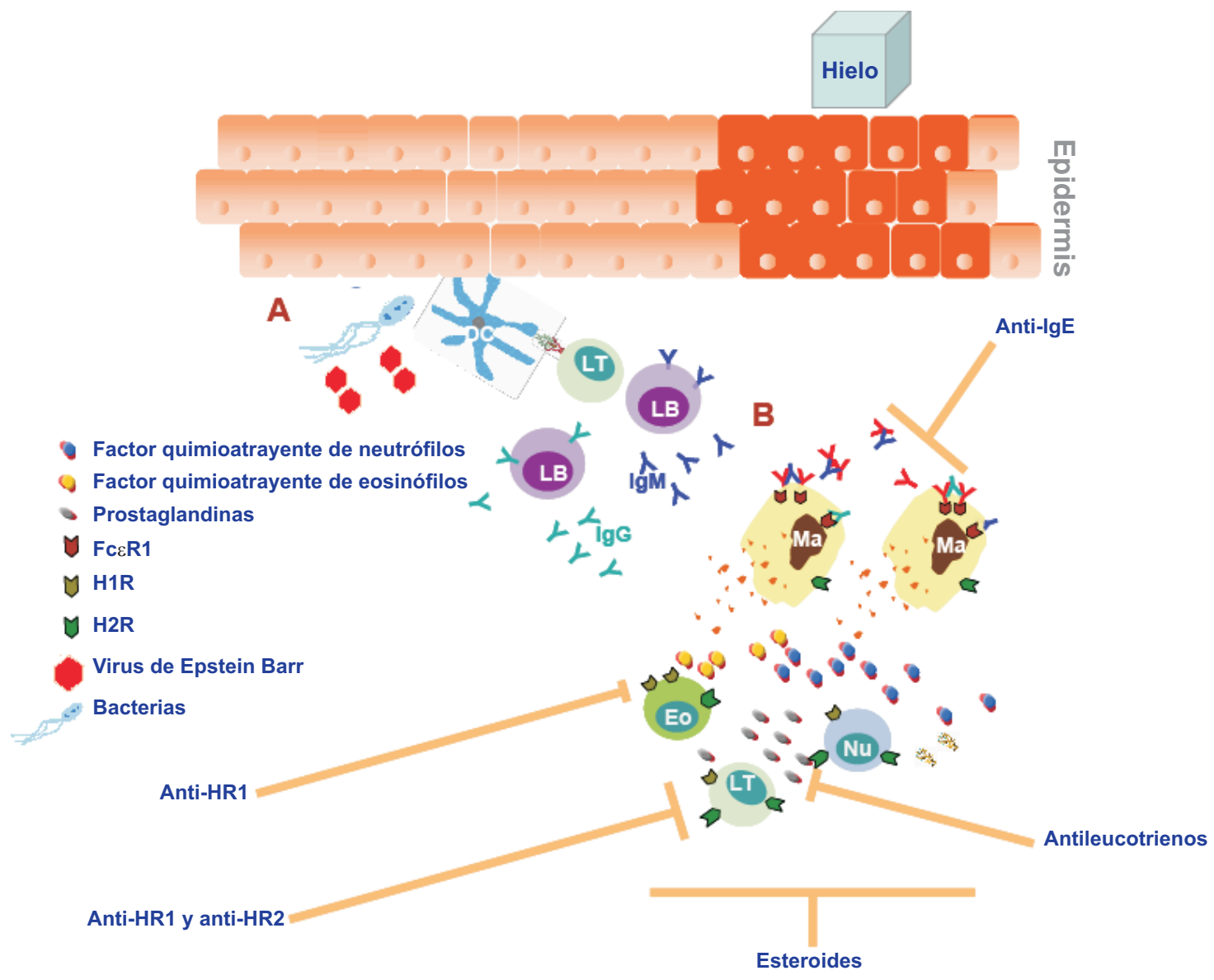

Figura 3. Fisiopatología y aproximaciones terapéuticas. Mecanismos de acción de la urticaria por frío y puntos de acción de los medicamentos.

(A) Formación de anticuerpos contra microorganismos que reconocen por mimetismo molecular, la lgE y el FcER1.

(B) Luego del reconocimiento de la lgG y la lgM del la lgE y el FcER se liberan varios mediadores de histamina que estimulan la respuesta inflamatoria, tanto celular como humoral.

producción elevada de $\lg \mathrm{E}$, como la picadura de un insecto (38), desarrollan con mayor frecuencia esta enfermedad que la población general y esto pudiera ser explicado en parte por este mecanismo. En estas situaciones se estimularía una mayor producción de lgG e lgM contra antígenos exógenos pero, por su parecido con los antígenos propios - en este caso, la lgE- reaccionarían contra ella estos últimos, incluso después de resuelta la infección y de eliminado el antígeno desencadenante.

Aunque estas hipótesis son sustentadas por experimentación in vitro y hallazgos in vivo, aún no se ha podido explicar cómo el frío promueve la interacción entre estas inmunoglobulinas y la $\operatorname{lgE}$. Lo que sí está más claro es que, cuando las inmunoglobulinas anti-lgE se unen a su blanco en la membrana de los mastocitos, se inicia la cascada inflamatoria que resulta en la liberación de la histamina y de otros mediadores (39). Estudios más recientes han demostrado que, además de los anticuerpos anti-lgE, también hay anticuerpos que reaccionan contra el receptor de alta afinidad de la IgE (Fc\&R1) en 30 a $40 \%$ de los pacientes con urticaria idiopática crónica y, posiblemente, en la urticaria por frío (40).

El complemento pudiera participar también en la respuesta inflamatoria del paciente con urticaria por frío, debido a que algunos subtipos de IgG (específicamente, el subtipo lgG1) tienen capacidad de activarlo (14). Esto ha sido respaldado en estudios practicados en algunos pacientes con urticaria secundaria por crioglobulinemia, pero faltan pruebas que apoyen su acción en la urticaria primaria adquirida. 
Debido a que los anti-anticuerpos están dirigidos contra la IgE y su receptor de alta afinidad (FceR1) que se encuentra principalmente en los mastocitos y los basófilos, es de esperar que estas células tengan un papel importante, principalmente los mastocitos, debido a que son los que se encuentran en mayor cantidad en el organismo y, en especial, en la piel. Los trabajos utilizando retos in vivo han demostrado el incremento de varios mediadores luego del reto con frío, tales como la histamina, la prostaglandina D2, el factor activador de plaquetas y los factor quimioatrayentes de neutrófilos y eosinófilos (41-43).

La participación de los eosinófilos, plaquetas y neutrófilos no ha sido estudiada a fondo, pero deben jugar un papel importante en la respuesta retardada. Cabe decir que, aunque muchos estudios muestran que la histamina es un mediador importante en la urticaria por frío, algunos estudios recientes señalan un papel menos relevante de esta molécula (44). Asero et al. observaron en cinco pacientes que con IgG baja mantenían la habilidad para liberar histamina; además, demostraron in vitro que la completa absorción de esta inmunoglobulina no modificaba la liberación de histamina; posteriormente, se demostró que IgG anti-lgE y anti-FceR1 no inducían liberación de histamina en ensayos de activación de mastocitos (44).

\section{Tratamiento}

El objetivo del tratamiento está enfocado en dos puntos. El primero es evitar reacciones graves que puedan comprometer la vida del paciente, lo cual se logra con medidas de evitación. En los pacientes con alto grado de sensibilidad al frío, es recomendable proporcionar adrenalina autoinyectable y esteroides para autoadministración en caso necesario, por el mayor riesgo de anafilaxia (45). El segundo punto del tratamiento es conseguir que el paciente logre tolerancia contra los estímulos fríos.

Para alcanzar el primer punto, se debe instruir al paciente para que evite los estímulos fríos; sin embargo, esta recomendación es difícil de llevar a cabo, por lo que las medidas terapéuticas actuales están enfocadas en el segundo objetivo.

La mayoría de los trabajos que buscan el mejor tratamiento se han hecho con grupos pequeños. Sin embargo, hay dos puntos claros en el manejo; primero, hay que evitar las exposiciones sistémicas a estímulos fríos, por ejemplo, bañarse en piscinas frías o duchas con agua fría, sobre todo cuando hay antecedentes de reacciones graves o la prueba del cubo del hielo es positiva en menos de tres minutos, porque tienen un mayor riesgo de anafilaxia. El segundo punto en el tratamiento es iniciar un manejo farmacológico que permita controlar los síntomas y que, además, se enfoque en prevenirlos.

Actualmente, la primera línea de manejo farmacológico que cumple estos objetivos son los antihistamínicos. El primer antihistamínico utilizado en urticaria por frío fue la ciproheptadina y, por ende, es el medicamento con el cual se tiene más experiencia (46). Actualmente, se le ha dado paso a otros antihistamínicos, como la cetirizina (47), la mizolastina (48), la loratadina, la desloratadina $(49,50)$, la ebastatina (51) y el ketotifeno (52). Aunque potencialmente todo antihistamínico antiH1R puede tener un efecto favorable, los de segunda generación tienen la ventaja de presentar menos efectos secundarios e igual efectividad (53), como lo observamos en el paciente del caso 1 con el uso de la fexofenadina. Todavía no hay estudios en los que se compare el efecto entre estos antihistamínicos en pacientes con urticaria por frío.

En pacientes que tengan manifestaciones graves se puede duplicar o incluso cuadruplicar la dosis diaria recomendada (54). En quienes el uso del antihistamínico no alcanza a controlar los síntomas, se puede adicionar un inhibidor del receptor de leucotrienos antileucotrieno (casos 2, 4 y 5); sin embargo, algunos resultados recientes cuestionan el efecto de los leucotrienos en la urticaria por frío (30).

En ocasiones, el cambio de antihistamínico puede producir mejoría clínica, como se observó en el paciente del caso 2. Otra opción sería el uso de un antiagregante plaquetario, como la rupatadina, que tiene un doble efecto antagonizando a la histamina e inhibiendo el facto activador de plaquetas $(55,56)$.

Se ha demostrado que la combinación de un antihistamínico $\mathrm{H} 1$ y $\mathrm{H} 2$ puede reportar algún beneficio, debido a que los anti-H2 pueden potenciar los efectos de los anti-H1, especialmente los que tienen metabolismo hepático, al disminuir la liberación de histamina por medio de la acción sobre el receptor H2 (H2R). Eso puede explicar el efecto positivo que ha demostrado tener la doxepina, debido a su actividad bloqueadora de los receptores $\mathrm{H} 1 \mathrm{R}$ que promueven la inflamación y a la agonista de los H2R que controlan la respuesta inflamatoria $(57,58)$. 
En los últimos años, el uso de anticuerpos monoclonales ha demostrado grandes beneficios en la medicina por permitir tratamientos específicos en diferentes enfermedades. Recientemente, se ha reportado el uso de omalizumab (anticuerpo anti-lgE) para el tratamiento de urticarias físicas aunque faltan estudios (59). También, faltan estudios controlados que demuestren su efectividad en los pacientes con urticaria por frío sin otras enfermedades alérgicas asociadas.

Los esteroides han demostrado tener alguna utilidad pero no se utilizan de rutina y se reservan para los pacientes que están expuestos de manera prolongada al frío o en quienes se demuestra una respuesta inflamatoria tardía al frío (60).

Hay que recordar que, en la mayoría de las ocasiones, esta enfermedad es autolimitada y dura 2 a 10 años luego del inicio de los síntomas. No se ha comprobado que las medidas farmacológicas propuestas logren acortar el tiempo de la enfermedad, pero muchos estudios demuestran mayor tolerancia ante la exposición al frío y reducción del riesgo de reaccionesgraves, lo cual se demuestra objetivamente reevaluando al paciente con la prueba del cubo de hielo después del inicio del tratamiento.

Lameta es lograr que tolere estímulos a tres minutos; sin embargo, puede ocurrir que la prueba resulte negativa por el tratamiento sin haber alcanzado aún el completo control clínico (caso 2). La mayoría de los reportes indican que el tratamiento reduce los síntomas y mejora la tolerancia al frío a los pocos días, hasta alcanzar una meseta que varía entre un paciente y otro. Cuando no se consigue este control, se recomienda inducir tolerancia al frío sumergiendo las extremidades del paciente (algunos autores recomiendan todo el cuerpo) en agua a $15{ }^{\circ} \mathrm{C}$ durante 5 minutos cada hora, hasta que desaparezca la urticaria de manera indefinida. $\mathrm{Si}$ el paciente suspende el tratamiento, puede volver a presentar los síntomas.

Para el caso de la urticaria familiar por frío, el manejo es básicamente el mismo que en las adquiridas. Otros medicamentos que están en estudio son los inmunomoduladores, como el interferón beta (29), antibióticos como la penicilina (4) o la capsaicina (61), que parecen ser algunas alternativas para pacientes con difícil mejoría; sin embargo, requieren ser estudiados más a fondo antes de recomendarlos de manera rutinaria.

\section{Conflicto de intereses}

Declaramos no tener conflicto de intereses.

\section{Financiación}

Esta publicación fue financiada por los autores.

\section{Referencias}

1. Siebenhaar F, Staubach P, Metz M, Magerl M, Jung J, Maurer M. Peltier effect-based temperature challenge: An improved method for diagnosing cold urticaria. J Allergy Clin Immunol. 2004;114:1224-5.

2. Kontou-Fili K, Borici-Mazi R, Kapp A, Matjevic LJ, Mitchel FB. Physical urticaria: Classification and diagnostic guidelines. Allergy. 1997;52:504-13.

3. Siebenhaar F, Weller K, Mlynek A, Magerl M, Altrichter S, Vieira Dos Santos R, et al. Acquired cold urticaria: Clinical picture and update on diagnosis and treatment. Clin Exp Dermatol. 2007;32:241-5.

4. Moller A, Henning M, Zuberbier T, Czarnetzki-Henz BM. Epidemiology and clinical aspects of cold urticaria. Hautarzt. 1996;47:510-4.

5. Zuberbier T, Bindslev-Jensen C, Canonica W, Grattan CE, Greaves MW, Henz BM, et al. EAACI/GA2LEN/EDF guideline: Definition, classification and diagnosis of urticaria. Allergy. 2006;61:316-20.

6. Konstantinou GN, Asero R, Maurer M, Sabroe RA, Schmid-Grendelmeier P, Grattan CE. EAACI/GA(2)LEN task force consensus report: The autologous serum skin test in urticaria. Allergy. 2009;64:1256-68.

7. Wanderer AA GK, Wasserman SI, Farr RS. Clinical characteristics of cold-induced systemic reactions in acquired cold urticaria syndromes: Recommendations for prevention of this complication and a proposal for a diagnostic classification of cold urticaria. J Allergy Clin Immunol. 1986;78:417.

8. Wanderer AA. Cold urticaria syndromes: Historical background, diagnostic classification, clinical and laboratory characteristics, pathogenesis, and management. J Allergy Clin Immunol. 1990;85:965-81.

9. Visitsuntorn N, Tuchinda M, Arunyanark N, Kerdsomnuk S. Ice cube test in children with cold urticaria. Asian Pac J Allergy Immunol. 1992;10:111-5.

10. Siebenhaar F, Staubach P, Metz M, Magerl M, Jung J, Maurer M. Peltier effect-based temperature challenge: An improved method for diagnosing cold urticaria. J Allergy Clin Immunol. 2004;114:1224-5.

11. Casale TB, Sampson HA, Hanifin J, Kaplan AP, Kulczycki A, Lawrence ID, et al. Guide to physical urticarias. J Allergy Clin Immunol. 1988;82:758-63.

12. Fernando SL. Cold-induced anaphylaxis. J Pediatr. 2009;154:148.

13. Alangari AA, Twarog FJ, Shih MC, Schneider LC. Clinical features and anaphylaxis in children with cold urticaria. Pediatrics. 2004;113:e313-7.

14. Wanderer AA, Hoffman HM. The spectrum of acquired and familial cold-induced urticaria/urticaria-like syndromes. Immunol Allergy Clin North Am. 2004;24:259-86.

15. Kivity S, Schwartz Y, Wolf R, Topilsky M. Systemic coldinduced urticaria -clinical and laboratory characterization. J Allergy Clin Immunol. 1990;85:52-4. 
16. Wanderer AA. Systemic cold urticaria (atypical acquired cold urticaria). J Allergy Clin Immunol. 1991;87:137-8.

17. Miller SD, Pritchard D, Crowley JP. Blood histamine levels following graded cold challenge in atypical acquired cold urticaria. Ann Allergy. 1992;68:27-9.

18. Kaplan AP. Unusual cold-induced disorders: Colddependent dermatographism and systemic cold urticaria. J Allergy Clin Immunol. 1984;73:453-6.

19. Kaplan AP, Garofalo J. Identification of a new physically induced urticaria: Cold-induced cholinergic urticaria. J Allergy Clin Immunol. 1981;68:438-41.

20. Back O, Larsen A. Delayed cold urticaria. Acta Derm Venereol. 1978;58:369-71.

21. Ting S, Mansfield LE. Localized cold-reflex urticaria. J Allergy Clin Immunol. 1985;75:421.

22. Czarnetzki BM, Frosch PJ, Sprekeler R. Localized cold reflex urticaria. Br J Dermatol. 1981;104:83-7.

23. Doeglas HM, Rijnten WJ, Schroder FP, Schirm J. Cold urticaria and virus infections: A clinical and serological study in 39 patients. Br J Dermatol. 1986;114:311-8.

24. Morais-Almeida M, Marinho S, Gaspar A, Arede C, Loureiro V, Rosado-Pinto J. Cold urticaria and infectious mononucleosis in children. Allergol Immunopathol (Madrid). 2004;32:368-71.

25. Lin RY, Schwartz RA. Cold urticaria and HIV infection. Br J Dermatol. 1993;129:465-7.

26. Miralles JC, Lopez FR, Sanchez-Gascon F, López C, Negro JM. Cold urticaria associated with acute serologic toxoplasmosis. Allergol Immunopathol (Madr). 2005;33:172-4.

27. Kranke B, Mayr-Kanhauser S, Aberer W. Helicobacter pylori in acquired cold urticaria. Contact Dermatitis. 2001;44:57-8.

28. Barranco P, Lopez C. Cold urticaria associated with serologic markers of hepatitis $B$ and cryoglobulinemia. Allergol Immunopathol (Madr). 1987;15:167-9.

29. Ito A, Kazama T, Ito K, Ito M. Purpura with cold urticaria in a patient with hepatitis $C$ virus infection-associated mixed cryoglobulinemia type III: Successful treatment with interferon-beta. J Dermatol. 2003;30:321-5.

30. Nuutinen P HI, Ackermann L. Histamine, but not leukotriene $\mathrm{C} 4$, is an essential mediator in cold urticaria wheals. Acta Derm Venereol. 2007;87:9-13.

31. Samsoe-Jensen T. Cold urticaria; report of a case: Passive transfer and in vitro experiments with skin cells. Acta Derm Venereol. 1955;35:107-10.

32. Sherman WB, Seebohm PM. Passive transfer of cold urticaria. J Allergy. 1950;21:414-24.

33. Wanderer AA, Maselli R, Ellis EF, Ishizaka K. Immunologic characterization of serum factors responsible for cold urticaria. J Allergy Clin Immunol. 1971;48:13-22.

34. Inoue S, Teshima H, Ago Y, Nagata S. Cold urticaria associated with immunoglobulin $\mathrm{M}$ serum factor. J Allergy Clin Immunol. 1980;66:299-304.

35. Newton HJ, Ang DK, van Driel IR, Hartland EL. Molecular pathogenesis of infections caused by Legionella pneumophila. Clin Microbiol Rev. 2010;23:274-98.
36. Honeyman MC, Stone NL, Falk BA, Nepom G, Harrison LC. Evidence for molecular mimicry between human $T$ cell epitopes in rotavirus and pancreatic islet autoantigens. $J$ Immunol. 2010;184:2204-10.

37. Arias-Santiago SA, Almazan-Fernández FM, BurkhardtPérez P, Naranjo-Sintes R. Cold urticaria associated with Epstein-Barr virus mononucleosis. Actas Dermosifiliogr. 2009;100:435-6.

38. Mathelier-Fusade $\mathbf{P}$, Leynadier F. Acquired cold urticaria after jellyfish sting. Contact Dermatitis. 1993;29:273.

39. Capulong MC, Tomikawa M, Tahara K, Akasawa A, likura Y. Cold stimulation test and histamine release in primary acquired cold urticaria. Int Arch Allergy Immunol. 1997;114:400-3.

40. Sabroe RA, Fiebiger E, Francis DM, Maurer D, Seed PT, Grattan CE, et al. Classification of anti-FcepsilonRI and anti-lgE autoantibodies in chronic idiopathic urticaria and correlation with disease severity. J Allergy Clin Immunol. 2002;110:492-9.

41. Wasserman SI, Soter NA, Center DM, Austen KF. Cold urticaria. Recognition and characterization of a neutrophil chemotactic factor which appears in serum during experimental cold challenge. J Clin Invest. 1977;60: 18996.

42. Grandel KE, Farr RS, Wanderer AA, Eisenstadt TC, Wasserman SI. Association of platelet-activating factor with primary acquired cold urticaria. $\mathrm{N}$ Engl $\mathrm{J}$ Med. 1985;313:405-9.

43. Rosenkranz AR, Wekkeli M, Hippmann G, Benda H, Jarisch R, Gotz M. Cold urticaria as a model of mediator release: Platelet factor 4, eosinophil cationic protein and histamine. Allergy. 1992;47:366-70.

44. Asero R, Tedeschi A, Lorini M. Histamine release in idiopathic cold urticaria. Allergy. 2002;57:1211-2.

45. Husz S, Tóth-Kása I, Kiss M, Dobozy A. Treatment of cold urticaria. Int J Dermatol. 1994;33:210-3.

46. Wanderer AA, St Pierre JP, Ellis EF. Primary acquired cold urticaria. Double-blind comparative study of treatment with cyproheptadine, chlorpheniramine, and placebo. Arch Dermatol. 1977;113:1375-7.

47. Bonadonna P, Lombardi C, Senna G, Canonica GW, Passalacqua G. Treatment of acquired cold urticaria with cetirizine and zafirlukast in combination. J Am Acad Dermatol. 2003;49:714-6.

48. Dubertret L, Pecquet C, Murrieta-Aguttes M, Leynadier F. Mizolastine in primary acquired cold urticaria. J Am Acad Dermatol. 2003;48:578-83.

49. Juhlin L. Inhibition of cold urticaria by desloratadine. J Dermatolog Treat. 2004;15:51-9.

50. Siebenhaar F, Degener F, Zuberbier T, Martus P, Maurer M. High-dose desloratadine decreases wheal volume and improves cold provocation thresholds compared with standard-dose treatment in patients with acquired cold urticaria: a randomized, placebo-controlled, crossover study. J Allergy Clin Immunol. 2009;123:672-9.

51. Magerl M, Schmolke J, Siebenhaar F, Zuberbier T, Metz M, Maurer M. Acquired cold urticaria symptoms can be safely prevented by ebastine. Allergy. 2007;62:1465-8. 
52. Visitsunthorn N, Tuchinda M, Vichyanond P. Cold urticaria in Thai children: Comparison between cyproheptadine and ketotifen in the treatment. Asian Pac J Allergy Immunol. 1995;13:29-35.

53. Villas F, Contreras FJ, López JM, López MC, Martínez F. A comparison of new nonsedating and classical antihistamines in the treatment of primary acquired cold urticaria (ACU). J Investig Allergol Clin Immunol. 1992;2:258-62.

54. Magerl M, Borzova E, Gimenez-Arnau A, Grattan CE, Lawlor F, Mathelier-Fusade $\mathbf{P}$, et al. The definition and diagnostic testing of physical and cholinergic urticarias--EAACI/GA2LEN/EDF/UNEV consensus panel recommendations. Allergy. 2009;64:1715-21.

55. Metz M, Scholz E, Ferrán M, Izquierdo I, Giménez-Arnau A, Maurer M. Rupatadine and its effects on symptom control, stimulation time, and temperature thresholds in patients with acquired cold urticaria. Ann Allergy Asthma Immunol. 2010;104:86-92.

56. Di Leo E, Nettis E, Cassano N, Foti C, Delle Donne P, Vena GA, et al. Treatment of acquired cold urticaria with rupatadine. Allergy. 2009;64:1387-8.
57. Neittaanmaki H, Myohanen T, Fraki JE. Comparison of cinnarizine, cyproheptadine, doxepin, and hydroxyzine in treatment of idiopathic cold urticaria: Usefulness of doxepin. J Am Acad Dermatol. 1984;11:483-9.

58. Neittaanmaki H, Fraki JE. Combination of localized heat urticaria and cold urticaria. Release of histamine in suction blisters and successful treatment of heat urticaria with doxepin. Clin Exp Dermatol. 1988;13:87-91.

59. Boyce JA. Successful treatment of cold-induced urticaria/ anaphylaxis with anti-lgE. J Allergy Clin Immunol. 2006;117:1415-8.

60. Black AK, Keahey TM, Eady RA, Greaves MW. Dissociation of histamine release and clinical improvement following treatment of acquired cold urticaria by prednisone. Br J Clin Pharmacol. 1981;12:327-31.

61. Toth-Kasa I, Jancso G, Obal F Jr, Husz S, Simon N. Involvement of sensory nerve endings in cold and heat urticaria. J Invest Dermatol. 1983;80:34-6. 